ARTICLE

\title{
Two-photon excited deep-red and near-infrared emissive organic co-crystals
}

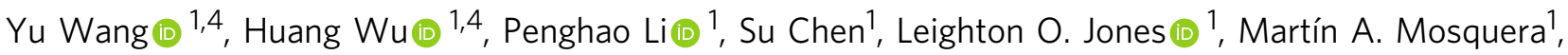 \\ Long Zhang1, Kang Cai (10 1, Hongliang Chen', Xiao-Yang Chen', Charlotte L. Stern (1) 1, \\ Michael R. Wasielewski (iD ${ }^{1}$, Mark A. Ratner ${ }^{1}$, George C. Schatz (iD ${ }^{1}$ \& J. Fraser Stoddart (iD ${ }^{1,2,3 凶}$
}

Two-photon excited near-infrared fluorescence materials have garnered considerable attention because of their superior optical penetration, higher spatial resolution, and lower optical scattering compared with other optical materials. Herein, a convenient and efficient supramolecular approach is used to synthesize a two-photon excited near-infrared emissive co-crystalline material. A naphthalenediimide-based triangular macrocycle and coronene form selectively two co-crystals. The triangle-shaped co-crystal emits deep-red fluorescence, while the quadrangle-shaped co-crystal displays deep-red and near-infrared emission centered on $668 \mathrm{~nm}$, which represents a $162 \mathrm{~nm}$ red-shift compared with its precursors. Benefiting from intermolecular charge transfer interactions, the two co-crystals possess higher calculated two-photon absorption cross-sections than those of their individual constituents. Their two-photon absorption bands reach into the NIR-II region of the electromagnetic spectrum. The quadrangle-shaped co-crystal constitutes a unique material that exhibits twophoton absorption and near-infrared emission simultaneously. This co-crystallization strategy holds considerable promise for the future design and synthesis of more advanced optical materials.

\footnotetext{
${ }^{1}$ Department of Chemistry, Northwestern University, 2145 Sheridan Road, Evanston, IL 60208, USA. ${ }^{2}$ School of Chemistry, University of New South Wales, Sydney, NSW 2052, Australia. ${ }^{3}$ Institute for Molecular Design and Synthesis, Tianjin University, 92 Weijin Road, Nankai District, Tianjin 300072, P.R. China.

${ }^{4}$ These authors contributed equally: Yu Wang, Huang Wu. ${ }^{凶}$ email: stoddart@northwestern.edu
} 
O rganic near-infrared (NIR) absorbing and emitting materials have found applications in fields as broad as bio-imaging ${ }^{1-3}$, photothermal therapy ${ }^{4-6}$, drug release ${ }^{7}$, night-vision technologies, and advanced optoelectronics ${ }^{8,9}$, for the simple reason that NIR light exhibits superior optical penetration, lesser photodamage, and lower optical scattering compared to visible light ${ }^{10-12}$. In order to fabricate NIR absorbing or emitting materials, several strategies are generally applied to tune the energy gap in these materials. They involve the extension of the length of $\pi$-conjugation in unsaturated molecules ${ }^{13}$ and the introduction of metal centers into coordination compounds ${ }^{8}$, as well as the association of electron-donor and acceptor units in molecular materials ${ }^{14,15}$. Moreover, two-photon absorption (TPA) materials, through the absorption of two photons simultaneously, are capable of converting the excitation wavelengths from the visible to the NIR region. This property engenders an alternative approach for the construction of NIR absorbing materials. Two-photon excited NIR-emitting materials, with inherent multifunctionality, are ideal candidates for cell imaging $^{16}$, photodynamic therapy ${ }^{17,18}$, microfabrication ${ }^{19}$, and optical data storage $e^{20,21}$. Consequently, the exploration of effective design strategies, along with the development of new twophoton excited NIR emissive materials, constitutes an attractive objective in materials science.

Recently, organic co-crystals, wherein donor and acceptor molecules assemble into highly ordered superstructures, based on intermolecular noncovalent bonding interactions, have emerged as a category of supramolecular materials with the following advantages ${ }^{22-24}$. (i) The preparation methods of co-crystals are similar to those of traditional single-component crystallization, which are feasible and easy to perform ${ }^{25}$. (ii) The properties of the co-crystals are related to their molecular packing and morphology, both of which can be modulated through co-crystal engineering without the requirement for further chemical synthesis ${ }^{26}$. (iii) Co-crystals not only maintain the properties of the individual components, but also, on some occasions, display unusual properties $^{27-29}$. For instance, a co-crystal based on tetrathiafulvalene (TTF) and tetracyanoquinodimethane (TCNQ) exhibits metallic electrical conductivity, in sharp contrast with the semiconductor characteristics of their individual crystals ${ }^{30}$. Although low molecular weight organic ferroelectrics are few and far between, charge transfer co-crystals, with lock-arm supramolecular ordering (LASO) motifs, exhibit ${ }^{31}$ ferroelectric behavior at room temperature. Inspired by these advances, extensive investigations of organic co-crystals have been pursued by scientists in different areas of materials science ${ }^{32,33}$. To date, abundant properties, such as ambipolar charge transport ${ }^{34}$, optical waveguide modulation ${ }^{35}$, piezochromic behavior ${ }^{36,37}$, thermal-mechanical response ${ }^{38}$, photothermal conversion ${ }^{39}$, and room-temperature phosphorescence ${ }^{40}$, have been achieved by simple co-crystallization. The engineering of NIR emissive cocrystals that exhibit TPA, however, remains as a challenge.

Additionally, the organic naphthalenediimide-based triangle (Fig. 1a and Supplementary Figs. 2-7), namely NDI- $\Delta^{41}$, consisting of three electron-deficient NDI units, linked by trans-1,2cyclohexano bridges, has been applied widely in supramolecular chemistry. On account of its well-defined inner cavity, NDI- $\Delta$ is capable $^{42}$ of undergoing selective assembly into either helical or non-helical nanotubes upon the encapsulation of halogenated solvent molecules, e.g., $\mathrm{XCH}_{2} \mathrm{CH}_{2} \mathrm{X}$ where $\mathrm{X}=\mathrm{Cl}, \mathrm{Br}$, I. The NDI$\Delta$, which is electron-deficient, is capable of encapsulating ${ }^{41}$ linear $\mathrm{I}_{3}-$ anions, resulting in the formation of single-handed helices in the solid state. Also, the electron-poor NDI- $\Delta$ and electron-rich TTF can, as a result of $[\pi \cdots \pi]$ interactions, co-assemble into twodimensional (2D) crystalline superstructures ${ }^{43}$. On the other hand, NDI- $\Delta$-based rechargeable lithium-ion batteries exhibit ${ }^{44}$ ultrahigh rate capability, as a result of the through-space electron sharing between NDI units in NDI- $\Delta$ molecules. The optical properties of NDI- $\Delta$ and its supramolecular assemblies, however, have hardly been investigated.

Herein, we examine a two-photon excited NIR emissive supramolecular material based on the co-crystallization of NDI- $\Delta$ and coronene (COR, Fig. 1b). Owing to the good match of their molecular sizes, the electron acceptor NDI- $\Delta$ and the electron donor COR can form two COR-NDI- $\Delta$ co-crystals (CNCs) with different morphologies - triangular and quadrangular in shapewhich have been denoted by CNC-T and CNC-Q, respectively. The selective co-crystallization can be controlled by varying both the nature of solvents and the donor-acceptor stoichiometries. Analyses of the solid-state superstructures reveal that CNC-Q exhibits a more efficient donor-acceptor $[\pi \cdots \pi]$ overlap compared with that of CNC-T, which engenders a stronger charge-transfer interaction in CNC-Q. Consequently, CNC-Q has a narrower bandgap and emits deep-red and NIR fluorescence centered on $668 \mathrm{~nm}$, while CNC-T displays deep-red fluorescence with a peak at $638 \mathrm{~nm}$. The TPA properties of NDI- $\Delta$ and COR are retained in the co-crystals. Benefiting from intermolecular charge-transfer interaction, CNC-T and CNC-Q possess higher TPA crosssections and longer two-photon excitation wavelengths that extend up into the NIR-II region when compared to their individual components. Overall, CNC-T and CNC-Q, not only constitute two rare co-crystals that exhibits TPA, as well as deep-red and NIR emission, but also serve as a powerful tool for uncovering the superstructure-property relationships. This investigation provides insights into tuning the optical properties in supramolecular systems and sheds light on the possibility of employing co-crystallization as a strategy for the production of multifunctional materials.

\section{Results}

Growth of NDI- $\Delta$ crystals and NDI- $\Delta$-based co-crystals. NDI- $\Delta$ molecules are known ${ }^{42}$ to adopt different packing arrangements in different solvents, indicating that their selection wields a significant influence on the crystallization of NDI- $\Delta$ molecules. In order to slow down the rate of crystal growth and obtain highquality crystals of $\mathrm{NDI}-\Delta, \mathrm{PhCl}$ was selected as the solvent because of its high boiling point. Upon slow evaporation of $\mathrm{PhCl}$, colorless one-dimensional (1D) needle NDI- $\Delta$ crystals were obtained (Supplementary Fig. 19). Single-crystal X-ray diffraction (XRD) analysis of the crystals reveals (Supplementary Fig. 11) that the NDI- $\Delta$ molecules stack in $1 \mathrm{D}$ columns, wherein adjacent molecules interact by means of $[\mathrm{C}-\mathrm{H} \cdots \mathrm{O}]$ hydrogen bonding between aromatic hydrogen and carbonyl oxygen atoms with distances ranging from 2.20 to $2.71 \AA$. Some $\mathrm{PhCl}$ molecules are included in the NDI- $\Delta$ columns, stabilized by a $[\mathrm{Cl} \cdots \pi]$ interaction with a distance of $3.37 \AA$ and $[\mathrm{C}-\mathrm{H} \cdots \pi]$ interactions covering distances of 2.66-2.90 ̊. The powder XRD pattern (Supplementary Fig. 18a) of the NDI- $\Delta$ crystals is consistent with the simulated pattern based on the single-crystal XRD data, indicating the high quality of the NDI- $\Delta$ crystals.

COR, an aromatic electron donor with good optoelectrical properties (Supplementary Fig. 21) ${ }^{45,46}$, possesses a strong ability ${ }^{38}$ to interact with electron acceptors. Owing to the larger size of COR compared to that of the NDI- $\Delta$ cavity, COR is only capable of interacting with the outer surface of NDI- $\Delta$ by means of $[\pi \cdots \pi]$ interactions. This mode of packing will increase the $[\pi \cdots \pi]$ overlap throughout the entire superstructure, enriching the potential optoelectrical properties of the co-crystals. Hence COR is employed to enter into co-crystallization with electronaccepting NDI- $\Delta$ molecules. High-quality crystals of COR could be grown (Supplementary Fig. 18b) by slow evaporation of its 


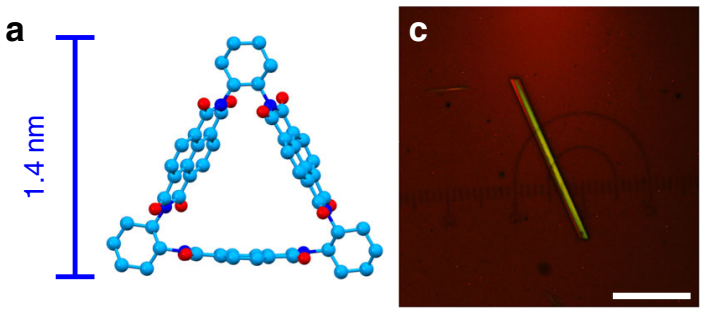

NDI- $\Delta$
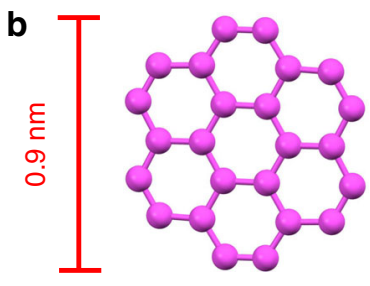

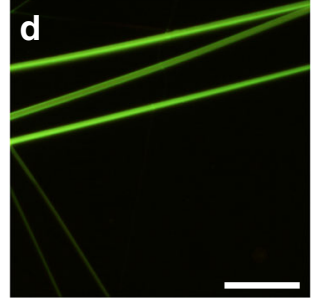

COR

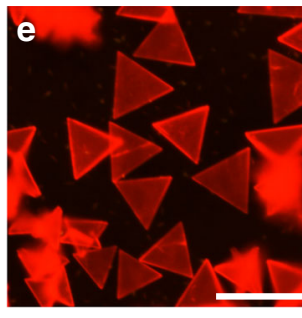

CNC-T

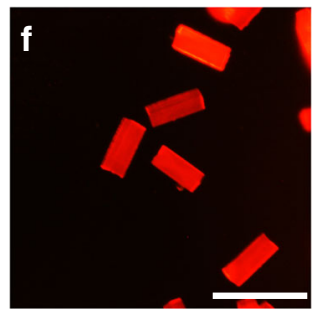

CNC-Q

Fig. 1 Crystal engineering of NDI- $\boldsymbol{\Delta}, \mathbf{C O R}, \mathbf{C N C}-\mathbf{T}$, and CNC-Q. a Solid-state structure of NDI- $\boldsymbol{\Delta}$. C light blue, N dark blue, O red. b Solid-state structure of COR. C pink. c Fluorescence microscopy image of an NDI- $\Delta$ crystal illustrating that it exhibits a 1D morphology and emits green fluorescence. Scale bar: $20 \mu \mathrm{m}$. d Fluorescence microscopy image of COR crystals, which display a 1D morphology and green fluorescence. Scale bar: $40 \mu \mathrm{m}$. e Fluorescence microscopy image of CNC-T, which exhibits a unique triangular morphology and red fluorescence. Scale bar: $50 \mu \mathrm{m}$. f Fluorescence microscopy image of CNC-Q, which displays a quadrangular morphology and red fluorescence. Scale bar: $50 \mu \mathrm{m}$.

$\mathrm{CH}_{2} \mathrm{Cl}_{2}$ solution. These crystals also exhibit (Supplementary Fig. 19) a $1 \mathrm{D}$ morphology.

The electron acceptor NDI- $\Delta$ and the COR donor, as a result of intermolecular charge transfer interactions, form (Supplementary Fig. 20) selectively two COR-NDI- $\Delta$ co-crystals (CNCs). One of them exhibits a unique triangular morphology, and the other possesses a quadrangular shape (Supplementary Fig. 19). Based on their morphologies, the two CNCs were named CNC-T and CNC-Q, respectively. The CNCs were found to be sensitive to the crystallization solvents and the respective donor-acceptor stoichiometries used in their preparation. For example, with a donor-acceptor molar ratio of 1:2, CNC-T can be obtained (Supplementary Fig. 18c) selectively by slow vapor diffusion of $\mathrm{MeOH}$ into a THF solution. Alternatively, with a donor-acceptor molar ratio of 1:1, CNC-Q can be obtained (Supplementary Fig. 18d) selectively by slow vapor diffusion of $\mathrm{Et}_{2} \mathrm{O}$ into a $\mathrm{CH}_{2} \mathrm{Cl}_{2}$ solution.

The fluorescence microscopy images reveal (Fig. 1c-f) that both CNC-T and CNC-Q emit bright red fluorescence, which is significantly different from the green fluorescence of individual NDI- $\Delta$ and COR crystals, indicating the influence of cocrystallization upon the optical behavior. With identical supramolecular co-constitutions but different donor-acceptor stoichiometries, CNC-T and CNC-Q serve as ideal models to unravel the superstructure-property relationships ${ }^{47}$ in these co-crystals. Meanwhile, reports relating to the influence of donor-acceptor stoichiometry on the luminescence behavior of co-crystals are rare $^{48,49}$. These facts motivated us to gain in-depth understanding to the superstructures and optical properties of CNC-T and CNC-Q.

Solid-state superstructures and morphologies of co-crystals. Single-crystal XRD analysis of CNC-T, in which the molar ratio between the donor and acceptor is 1:2, reveals that it belongs (Supplementary Table 3) to the monoclinic C2 space group. NDI$\Delta$ and COR adopt (Fig. 2a) a face-to-face stacking mode based on $[\pi \cdots \pi]$ and $[\mathrm{C}-\mathrm{H} \cdots \pi]$ interactions. The plane-to-plane distances of the donor-acceptor $[\pi \cdots \pi]$ interactions between the COR and one of the NDI units range from 3.24 to $3.38 \AA$. The $[\mathrm{C}-\mathrm{H} \cdots \pi]$ interactions refer to (Supplementary Fig. 13) the cyclohexano hydrogen atoms in NDI- $\Delta$ and the plane of COR with distances ranging from 2.60 to $2.88 \AA$. The donor-acceptor pairs of COR and NDI- $\Delta$ stack in a $1 \mathrm{D}$ columnar superstructure (Fig. 2c), through $[\mathrm{C}-\mathrm{H} \ldots \mathrm{O}]$ hydrogen bonding between aromatic hydrogen and carbonyl oxygen atoms in two adjacent NDI- $\Delta$ molecules (Fig. 2b) with distances ranging from 2.31 to $2.59 \AA$. These $1 \mathrm{D}$ columns pack into a 2D layer in the $a-b$ plane (Supplementary Fig. 14c) by means of $[\mathrm{C}-\mathrm{H} \cdots \pi]$ interactions (Supplementary Fig. 13) between cyclohexano hydrogen atoms and the NDI plane in neighboring NDI- $\Delta$ molecules with distances in the range of 2.69-2.90 A. The 2D layers are tightly packed (Fig. $2 \mathrm{~d}$ ) along the $c$ axis and form a well-ordered three-dimensional (3D) array.

CNC-T displays a triangular morphology, an observation which is not common in organic crystals. In order to gain more insight into the formation of this geometry, powder XRD analysis and morphology simulation were carried out on CNC-T. The powder XRD pattern of CNC-T, grown on a glass substrate, displays (Supplementary Fig. 18c) strong (001) and (002) diffraction peaks, indicating ${ }^{35}$ that the triangular growth face of the co-crystal is parallel to the (001) lattice plane. This experimental data is confirmed by the simulated morphology of the co-crystals. CNC-T possesses (Supplementary Fig. 14) an isosceles triangular geometry, wherein the (001) and (00-1) growth faces account for (Supplementary Table 1) the largest facet area percentage of $42.5 \%$ as a result of their lowest attachment energy $\left(E_{\text {att }}\right)$. The vertex angle of a simulated isosceles triangle is $62^{\circ}$, an angle which is consistent with that $\left(62^{\circ}\right)$ between the (110) and (1-10) lattice planes observed (Supplementary Fig. 15) in the single-crystal XRD data. Benefiting from the high symmetry in the $C 2$ space group, the (110) and (1-10) growth faces display identical $E_{\text {att }}$ and the exposed facet area percentage in the simulated morphology, so that CNC-T adopts an isosceles triangular geometry.

In contrast, CNC-Q, with a 1:1 donor-acceptor stoichiometry, crystallizes (Supplementary Table 3 ) in a triclinic $P 1$ space group. With the change in donor-acceptor ratio, NDI- $\Delta$ molecules are surrounded (Fig. 3a) by one more equivalent of COR molecules in CNC-Q than that observed in CNC-T. This stoichiometry 

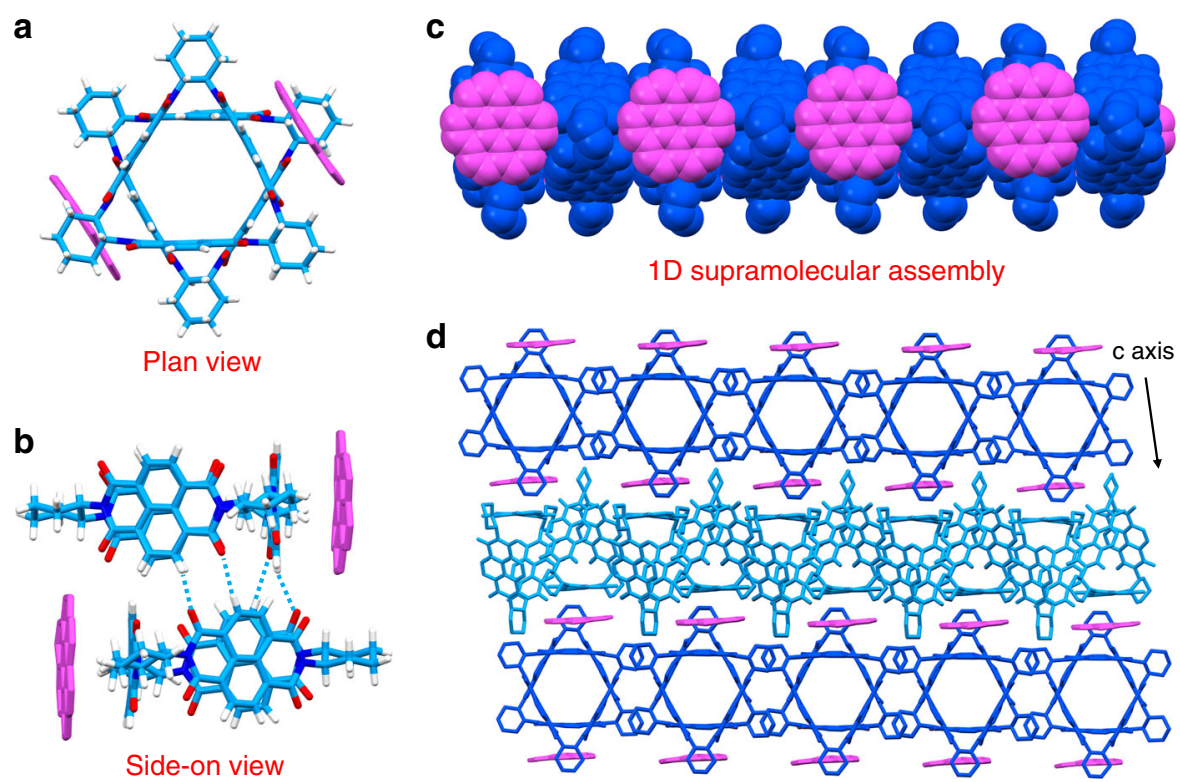

Fig. 2 Solid-state superstructure of CNC-T. a Plan view of a capped-sticks representation demonstrating the face-to-face packing between COR and

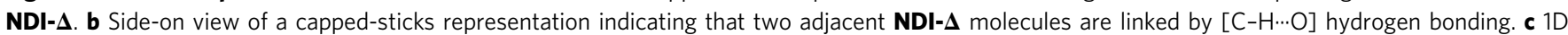
Columnar superstructure composed of inner NDI- $\boldsymbol{\Delta}$ nanotube and outer COR molecules. $\mathbf{d}$ Solid-state superstructure of CNC-T illustrating the layer-bylayer stacking along the $c$ axis.

variation, along with the through-space electron sharing between NDI units in the NDI- $\Delta^{41}$ (Supplementary Fig. 8), results in CNC-Q exhibiting a more efficient donor-acceptor overlap. In the solid-state superstructure of CNC-Q, NDI- $\Delta$ and COR molecules also exhibit (Supplementary Fig. 16a) face-to-face stacking stabilized by $[\pi \cdots \pi]$ and $[\mathrm{C}-\mathrm{H} \cdots \pi]$ interactions with distances of $3.29-3.39$ and $2.74-2.90 \AA$, respectively. The 1D columnar supramolecular assembly, composed of an inner NDI$\Delta$ nanotube and a layer of outer COR molecules, is also formed (Fig. $3 \mathrm{~b}, \mathrm{c}$ ) along the $a$ axis, supported by $[\mathrm{C}-\mathrm{H} \ldots \mathrm{O}]$ hydrogen bonds with distances ranging from 2.40 to $2.60 \AA$. These $1 \mathrm{D}$ colunms stack (Fig. 3d) in 2D layers in the $a-b$ plane (Supplementary Fig. 17d) interconnected by COR molecules through (Supplementary Fig. 16a) $[\pi \cdots \pi]$ and $[\mathrm{C}-\mathrm{H} \cdots \pi]$ interactions. Subsequently, these 2D layered nanostructures are held together by coaxial NDI- $\Delta$ dimers (Supplementary Fig. 16b), relying on $[\pi \cdots \pi]$ interactions, to form (Fig. $3 \mathrm{~d}$ ) well-ordered $3 \mathrm{D}$ arrays along the $c$ axis.

Differing from the triangular morphology of CNC-T, CNC-Q exhibits a quadrangular geometry with an angle of $95^{\circ}$. The value is the same as that observed (Supplementary Fig. 17) from the $(011)$ and $(0-1-1)$ growth faces in the simulated morphology of CNC-Q. According to the theoretical calculations, the $E_{\text {att }}$ of $(001)$ and $(00-1),(011)$ and $(0-1-1),(100)$ and $(-100)$ growth faces are (Supplementary Table 2) $-64.3,-66.4$, and $-81.0 \mathrm{kcal} /$ mol, respectively. These energies lead to the percentages of their exposed facet areas in the simulated morphology being 34.6, 33.6, and $26.8 \%$, respectively, explaining why we observe the quadrangle-shaped CNC-Q.

In summary, comparing these two co-crystals, we can conclude that (i) NDI- $\Delta$ and COR prefer to adopt a face-to-face packing motif driven by charge-transfer interactions even in different solvents, (ii) NDI- $\Delta$ is prone to assemble into $1 \mathrm{D}$ supramolecular arrays supported by multiple [C-H...O] hydrogen bonds, and (iii) alteration in donor-acceptor stoichiometries results in dramatic differences in the solid-state superstructures and morphologies.
Absorption and fluorescence spectra. In order to elucidate the photophyscial properties of CNC-T and CNC-Q, solid-state UV$\mathrm{Vis}$ absorption and fluorescence spectra were recorded. NDI- $\Delta$ crystals show (Fig. 4a) strong absorption in the region of 240-420 $\mathrm{nm}$, and COR crystals absorb primarily at $240-450 \mathrm{~nm}$. Distinctive absorption spectra were observed after co-crystallization. CNC-T exhibits a broad absorption ranging from 240 to $596 \mathrm{~nm}$, which is significantly red-shifted in comparison with the individual NDI- $\Delta$ and COR crystals, on account of the intermolecular charge-transfer interaction in the co-crystal. By contrast, the absorption band of CNC-Q extends up to $617 \mathrm{~nm}$, which is $21 \mathrm{~nm}$ red-shifted compared to that of CNC-T. This difference may arise from a more efficient donor-acceptor $[\pi \cdots \pi]$ overlap in the solidstate superstructure of CNC-Q than in that of CNC-T.

Fluorescence spectra reveal (Fig. 4b) that the two co-crystals display a red-shifted emission compared to that of the individual molecular crystals, an observation which is in agreement with the fluorescence microscopy images (Fig. 1). NDI- $\Delta$ and COR crystals show green fluorescence with emission peaks centered on 488 and $506 \mathrm{~nm}$, respectively. Their corresponding solid-state photoluminescence quantum yields (PLQYs, $\Phi_{F}$ ) are 1.4 and $3.2 \%$. In comparison with their solution-state fluorescence spectra (Supplementary Fig. 21), the solid-state fluorescence spectra of NDI- $\Delta$ and COR crystals are red-shifted, on account of their particular packing modes in the solid state ${ }^{50}$. NDI- $\Delta$ molecules have a rigid triangular geometry, which prevents the formation of $\mathrm{H}$-type aggregates, while COR molecules adopt (Supplementary Fig. 12) a slipped face-toface packing mode with J-type aggregation in their crystalline state. CNC-T exhibits (Fig. 4b) deep-red fluorescence ${ }^{51}$ with a peak at $638 \mathrm{~nm}$, while CNC-Q displays (Fig. 4b) deep-red and NIR fluorescence with a maximum emission wavelength at $668 \mathrm{~nm}$, which is red-shifted by $162 \mathrm{~nm}$ compared to that of the COR crystals. The solid-state PLQYs of CNC-T and CNC-Q are 0.9 and $2.2 \%$, respectively, values which are comparable to those of the individual component crystals. These observations underline the viability of the co-crystallization strategy in producing solidstate NIR functional materials ${ }^{11,52}$. Time-resolved fluorescence 


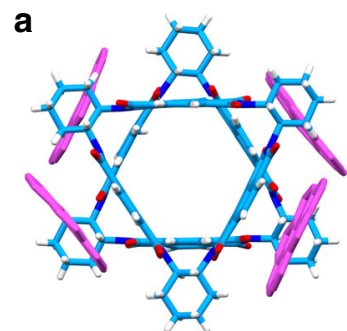

Plan view

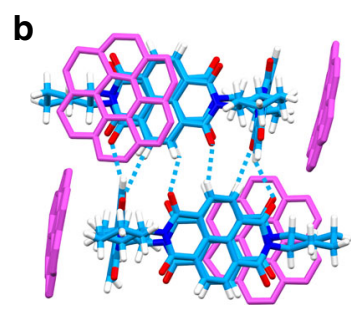

Side-on view

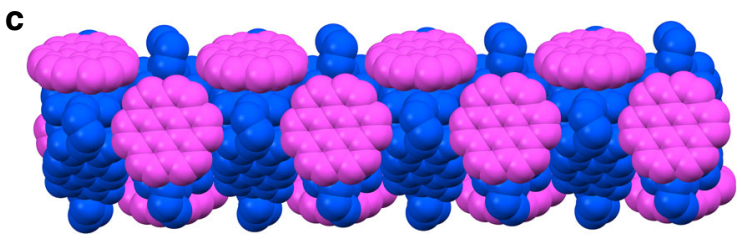

1D supramolecular assembly

d

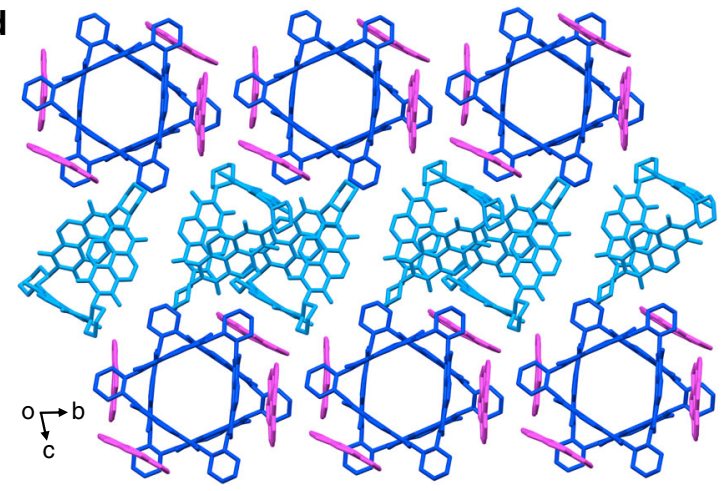

Fig. 3 Solid-state superstructure of CNC-Q. a Plan view of a capped-sticks representation illustrating that NDI- $\mathbf{\Delta}$ and $\mathbf{C O R}$ molecules adopt a face-to-face

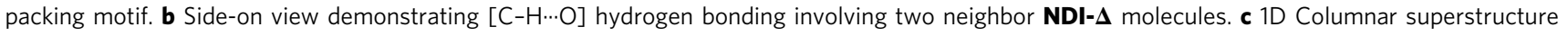
composed of inner NDI- $\boldsymbol{\Delta}$ nanotube and outer $\mathbf{C O R}$ molecules. $\mathbf{d}$ Solid-state superstructure of $\mathbf{C N C}-\mathbf{Q}$ revealing the layer-by-layer stacking along the $c$ axis.
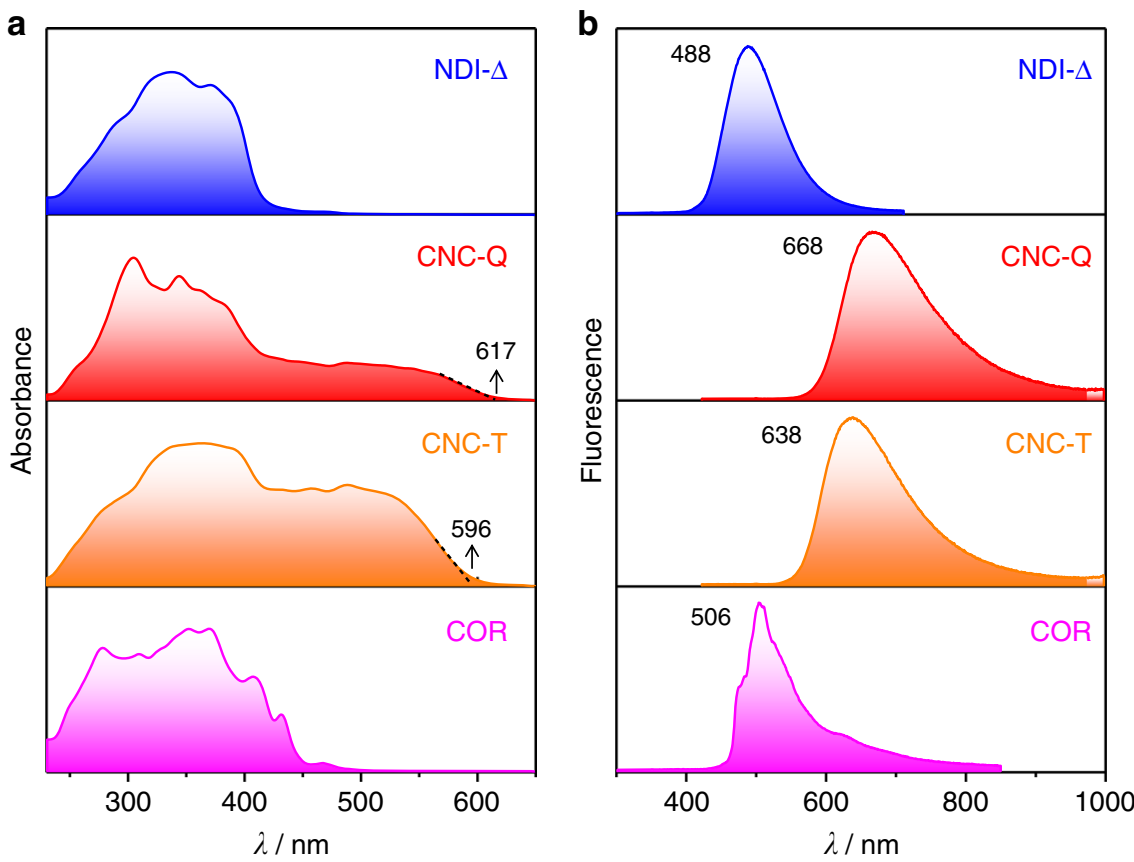

Fig. 4 Solid-state spectroscopic characterization. Solid-state a UV-Vis absorption and b fluorescence spectra of NDI- $\mathbf{\Delta}, \mathbf{C N C}-\mathbf{Q}, \mathbf{C N C}-\mathbf{T}$ and $\mathbf{C O R}$. The spectra of CNC-T and CNC-Q are significantly red-shifted compared to those of NDI- $\mathbf{\Delta}$ and COR crystals.

measurements reveal that both CNC-T and CNC-Q exhibit (Supplementary Fig. 22) a double-exponential fluorescence decay curve with an average lifetime of 11.4 and $9.7 \mathrm{~ns}$, respectively. The radiative rate constant $\left(k_{\mathrm{r}}\right)$ and non-radiative rate constant $\left(k_{\mathrm{nr}}\right)$ of CNC-T are (Supplementary Table 4) $7.9 \times 10^{5}$ and $8.7 \times 10^{7} \mathrm{~s}^{-1}$, respectively, while those for CNC-Q are $2.3 \times 10^{6}$ and $1.0 \times 10^{8} \mathrm{~s}^{-1}$. The larger $k_{\mathrm{r}}$ and $k_{\mathrm{nr}}$ values of $\mathbf{C N C}-\mathbf{Q}$ are consistent with its faster fluorescence decay lifetime when compared with that of CNC-T. These observations indicate that the photophysical properties of cocrystals are sensitive to their solid-state superstructures. On account of the different donor-acceptor stoichiometry and the through-space electron sharing between NDI units in the NDI- $\Delta^{41}$ (Supplementary Fig. 8), CNC-Q exhibits more efficient donor-acceptor overlap and stronger intermolecular chargetransfer interactions compared with CNC-T. Consequently, CNC$\mathbf{Q}$ displays the more red-shifted absorption and emission spectra.

Frontier molecular orbitals calculations. In order to attain a deeper understanding of the electronic structures of the cocrystals, density functional theory (DFT) calculations were conducted (Supplementary Figs. 31-34) with the B3LYP functional 

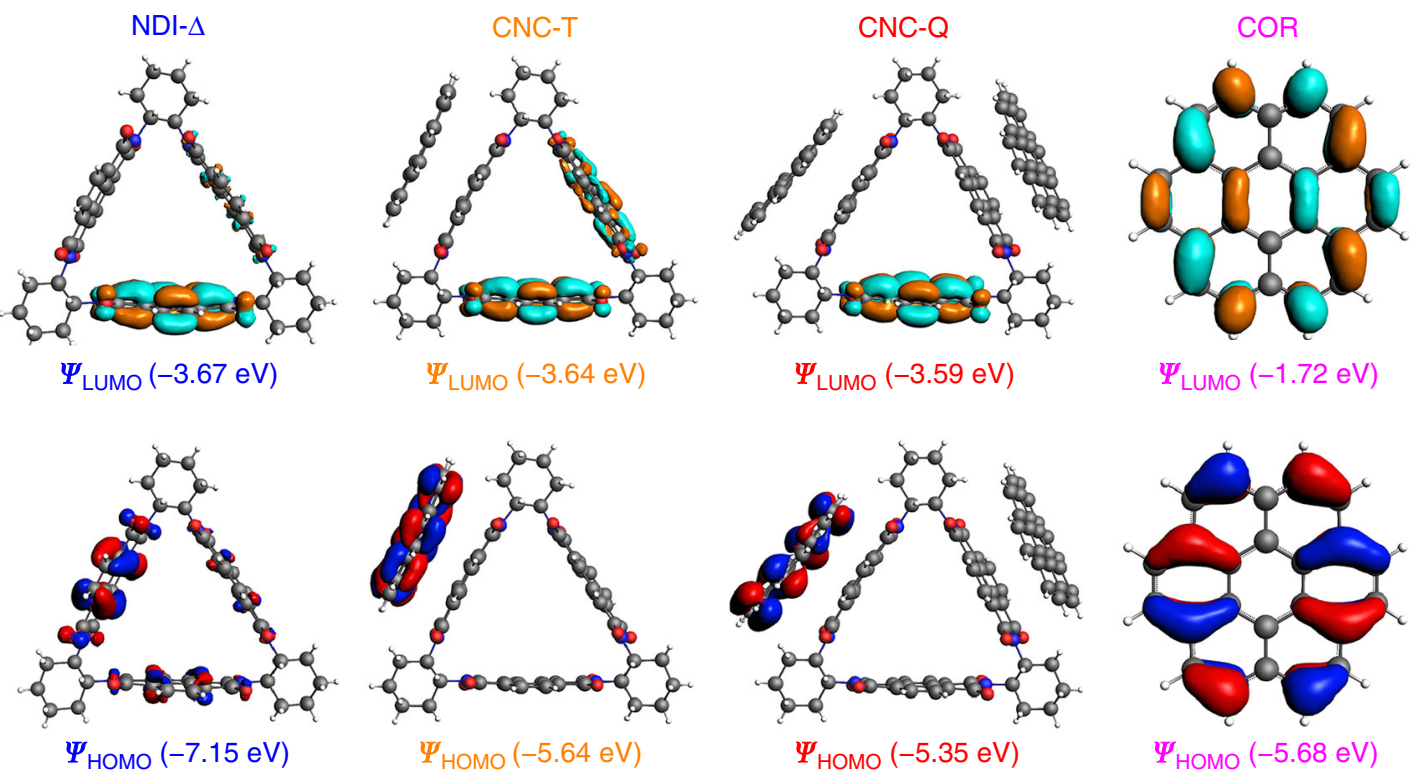

Fig. 5 DFT Calculations. Frontier molecular orbitals of NDI- $\mathbf{A}, \mathbf{C N C}-\mathbf{T}, \mathbf{C N C}-\mathbf{Q}$ and $\mathbf{C O R}$ along with their corresponding energy gaps, which are 3.47, 2.00, 1.76 and $3.97 \mathrm{eV}$, respectively.

and an all-electron triple zeta basis set. The frontier molecular orbital diagrams demonstrate (Fig. 5) that the highest occupied molecular orbitals (HOMOs) of CNC-T and CNC-Q are concentrated on the electron-donating COR molecule with energies of -5.64 and $-5.35 \mathrm{eV}$, respectively. These values are close to that of COR $(-5.68 \mathrm{eV})$. The lowest unoccupied molecular orbitals (LUMOs) of CNC-T and CNC-Q are localized on the electronaccepting NDI- $\Delta$ molecule with energies of -3.64 and $-3.59 \mathrm{eV}$, respectively, resembling that of NDI- $\Delta(-3.67 \mathrm{eV})$. These observations indicate that the HOMO of co-crystal is primarily derived from the HOMO of the donor, while the LUMO of cocrystal is chiefly dependent on that of the acceptor. The calculated HOMO-LUMO energy gaps $(\Delta E)$ of CNC-T and CNC-Q are 2.00 and $1.76 \mathrm{eV}$, respectively. This tendency is in agreement with the results obtained $(\Delta E=2.09$ and $2.01 \mathrm{eV}$ for CNC-T and CNC-Q, respectively) from UV-Vis absorption spectra. The reason for the narrower bandgap of CNC-Q arises from the change of donor-acceptor ratio from 1:2 in CNC-T to 1:1 in CNC-Q. The results demonstrate that the stoichiometric variation leads to the changes in supramolecular arrangements, hence tuning the electronic structures and photophysical properties of the co-crystals.

Two-photon absorption. TPA is a third-order nonlinear optical process, which occurs upon exposure of materials to highintensity lasers. Upon excitation by a $700 \mathrm{~nm}$ laser, NDI- $\Delta$ crystals were observed (Fig. 6a) by a two-photon microscope. Their emission was collected in the green detection channel (detection region: $470-550 \mathrm{~nm}$ ), corresponding closely to their fluorescence emission $(488 \mathrm{~nm})$. Similar fluorescence emission by NDI- $\Delta$ crystals can also be detected (Fig. $6 \mathrm{~b}-\mathrm{d}$ ) in the green channel of the two-photon microscope when excited at 800, 900, and $1000 \mathrm{~nm}$. These observations indicate that NDI- $\Delta$ crystals are capable of emitting light at a shorter wavelength upon excitation at a longer wavelength, a process which corresponds to photon upconversion. When the excitation wavelength is fixed at $740 \mathrm{~nm}$, the fluorescence intensity of NDI- $\Delta$ crystals is proportional to the square of the incident laser power (Fig. 7a and Supplementary Fig. 23), implying that the upconversion emission of NDI- $\Delta$ crystals originates from a TPA process ${ }^{53}$. COR crystals display a similar TPA process (Fig. $6 \mathrm{e}-\mathrm{h}$ ) to that of NDI- $\Delta$ crystals. Upon excitation at $700,800,900$, and $1000 \mathrm{~nm}$, the upconversion emission by COR crystals, which agrees with their fluorescence emission $(506 \mathrm{~nm})$, is also collected in the green detection channel. At a fixed excitation wavelength of $740 \mathrm{~nm}$, the fluorescence intensity of COR crystals increases (Fig. 7b and Supplementary Fig. 24) linearly with the square of laser power, indicating an active TPA process.

Similarly, the two co-crystals also exhibit nonlinear optical responses. At laser irradiation from 700 to $1000 \mathrm{~nm}$, the upconversion emission of CNC-T is collected (Fig. 6i-1) in the red detection channel (detection region: 550-650 nm), which corresponds closely to the fluorescence emission $(638 \mathrm{~nm})$ of CNC-T. At a fixed excitation wavelength of $1000 \mathrm{~nm}$, the fluorescence intensity of CNC-T displays (Fig. 7c and Supplementary Fig. 25) a linear dependence on the square of the laser power. The TPA of CNC-Q is also detected (Fig. 6m-p) by two-photon imaging in the red channel, in accordance with its fluorescence emission band in the region of $580-950 \mathrm{~nm}$. The corresponding excitation power dependence of fluorescence intensity is illustrated in Fig. $7 \mathrm{~d}$ and Supplementary Fig. 26. In addition, the TPA of the co-crystals is further supported by time-dependent densityfunctional theory (TDDFT) calculations (Supplementary Fig. 35 and Table 6). In the calculated one-photon absorption spectra, the lowest-energy excited state of CNC-T corresponds to the wavelength of $677 \mathrm{~nm}$ with a negligible oscillator strength of 0.009 a.u.. CNC-Q exhibits three lowest-energy excitations at the wavelength of 725,770 , and $827 \mathrm{~nm}$, with quite small oscillator strengths of $0.027,0.001$, and 0.020 a.u., respectively. Consequently, the emission of the co-crystals at excitation wavelengths of $\sim 1000 \mathrm{~nm}$ stems mainly from the TPA rather than the one-photon absorption of their lowest-energy excited states.

Taking into consideration that the TPA is governed by different quantum-mechanical rules, in comparison with onephoton absorption, the TPA spectra can display distinct line shapes compared to their one-photon spectra ${ }^{54}$. The two-photon excitation spectra of these crystals were recorded to explore the excitation wavelength dependence of their upconversion emission intensity (Supplementary Figs. 27-30). For this purpose, an in-situ two-photon imaging experiment was conducted at a constant laser power, while the laser excitation wavelengths were varied in the range of $700-1000 \mathrm{~nm}$ by intervals of $20 \mathrm{~nm}$. 
$\lambda_{\mathrm{ex}}=700 \mathrm{~nm}$
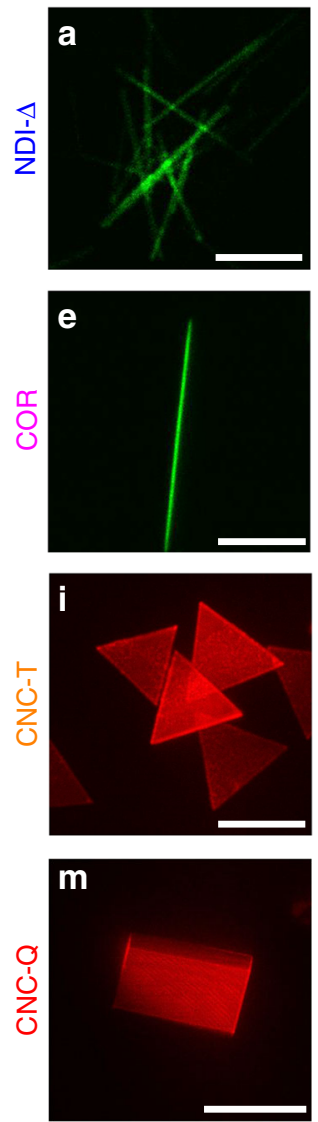

$\lambda_{\mathrm{ex}}=800 \mathrm{~nm}$
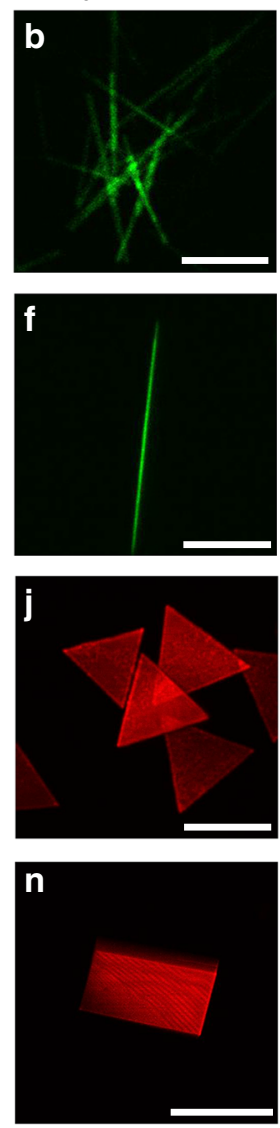

$\lambda_{\mathrm{ex}}=900 \mathrm{~nm}$
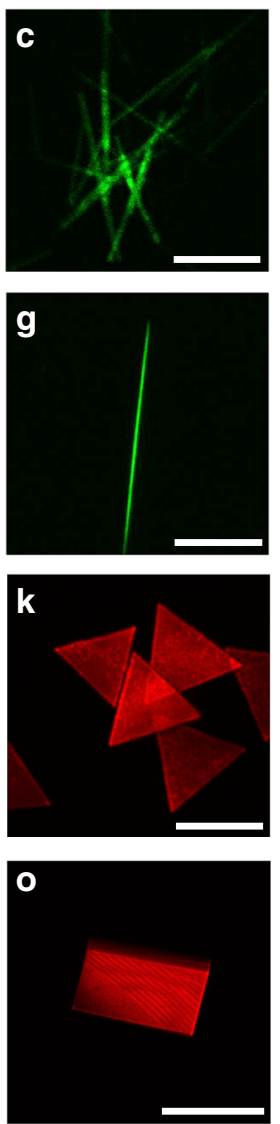

$\lambda_{\mathrm{ex}}=1000 \mathrm{~nm}$
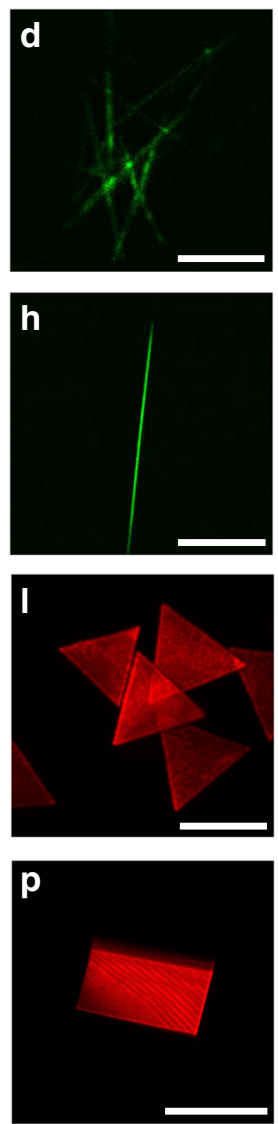

Fig. 6 Photon upconversion characteristics. Two-photon microscopy images of $\mathbf{a}-\mathbf{d} \mathbf{~ N D I}-\mathbf{\Delta}, \mathbf{e}-\mathbf{h} \mathbf{C O R}, \mathbf{i}-\mathbf{I} \mathbf{C N C}-\mathbf{T}$ and $\mathbf{~} \mathbf{m}-\mathbf{p}$ CNC-Q. a-d The excitation laser powers for NDI- $\mathbf{\Delta}$ crystals were $7.0,30.4,54.0$, and $54.0 \mathrm{~mW}$, respectively. Scale bar: $20 \mu \mathrm{m}$. $\mathbf{e}-\mathbf{h}$ The excitation laser powers for COR crystal were 6.7, 37.1, 72.0, and $54.0 \mathrm{~mW}$, respectively. Scale bar: $100 \mu \mathrm{m}$. i-I The excitation laser powers for CNC-T were 6.3, 8.1, 3.5, and 2.7 mW, respectively. Scale bar: $40 \mu \mathrm{m} . \mathbf{m}-\mathbf{p}$ The excitation laser powers for CNC-Q were 3.0, 3.8, 1.6, and $1.1 \mathrm{~mW}$, respectively. Scale bar: $100 \mu \mathrm{m}$.

The upconversion emission intensity was collected from the same sample region and then plotted as the function of the excitation wavelength. The resulting two-photon excitation spectra (Fig. 8) reveal that NDI- $\Delta$ and COR crystals display the strongest upconversion fluorescence at $700 \mathrm{~nm}$. In sharp contrast, the twophoton excitation spectra of CNC-T and CNC-Q exhibit a similar shape with peaks centered on $980 \mathrm{~nm}$. These profiles indicate that co-crystallization leads to a red-shifted TPA spectrum compared to the individual molecular crystals. Remarkably, CNC-Q serves as a co-crystal that exhibits two-photon response and NIR emission simultaneously, implying that the co-crystallization strategy serves as a convenient and efficient design approach towards multifunctional optical materials.

Two-photon excitation spectra simulations. Second linear response TDDFT calculations were performed using an in-house code to gain a theoretical understanding of the TPA spectrum $^{55,56}$. The calculated TPA spectra (Fig. 9) of all four materials are consistent with the experimental results (Fig. 8). The NDI- $\Delta$ crystals display a broad TPA spectrum in the region of $600-800 \mathrm{~nm}$, and exhibit a maximum TPA cross-section $\left(\sigma_{\mathrm{TPA}}\right)$ of $60 \mathrm{GM}$ at $690 \mathrm{~nm}$. The COR crystals display a sharp twophoton excitation peak at $658 \mathrm{~nm}$ with a weak $\sigma_{\mathrm{TPA}}$ of $8.8 \mu \mathrm{GM}$. The maximum $\sigma_{\mathrm{TPA}}$ of CNC-T and CNC-Q are $1939 \mathrm{GM}$ at 643 $\mathrm{nm}$ and $256 \mathrm{GM}$ at $624 \mathrm{~nm}$, respectively. These values are 32 and 4.3 times larger than that for NDI- $\Delta$ crystals. CNC-T possesses higher $\sigma_{\mathrm{TPA}}$ than that for $\mathbf{C N C}-\mathbf{Q}$, a difference which may arise from the fourth-power dependency of $\sigma_{\mathrm{TPA}}$ on the transition dipoles and the average values of transition dipoles for CNC-T are $48 \%$ higher than those for CNC-Q. Moreover, in the calculated TPA spectra of the co-crystals, a new absorption band was identified in the range from 1000 to $1300 \mathrm{~nm}$ in the NIR-II region $^{57,58}$, with a maximum $\sigma_{\mathrm{TPA}}$ of $92 \mathrm{GM}$ at $1290 \mathrm{~nm}$ and 65 GM at $1177 \mathrm{~nm}$ for CNC-T and CNC-Q, respectively. The possible reasons for the increased TPA cross-sections and red-shifted TPA spectra of co-crystals include the following. (i) The donor and acceptor molecules adopt a face-to-face packing motif, facilitating the $[\pi \cdots \pi]$ overlap throughout the entire superstructure. (ii) The intermolecular charge-transfer interaction induces the $\pi$-electron delocalization from the donor to the acceptor molecules, contributing to the supramolecular electronic polarization in the co-crystals ${ }^{53,59}$. These results imply that the design of charge transfer co-crystals emerges as an alternative approach for the synthesis of TPA materials with enhanced twophoton response. In the rational design of two-photon excited NIR emissive co-crystals, two fundamental points can be taken into consideration-namely, selecting TPA-active precursors in order to induce nonlinear optical properties, and introducing intermolecular charge-transfer interactions in order to achieve NIR emission and enhanced two-photon responses.

\section{Discussion}

We have applied a supramolecular approach to the design of twophoton excited deep-red and NIR emissive materials based on the co-crystallization of NDI- $\Delta$ and COR. Two charge transfer cocrystals, CNC-T and CNC-Q, have been obtained by controlling 

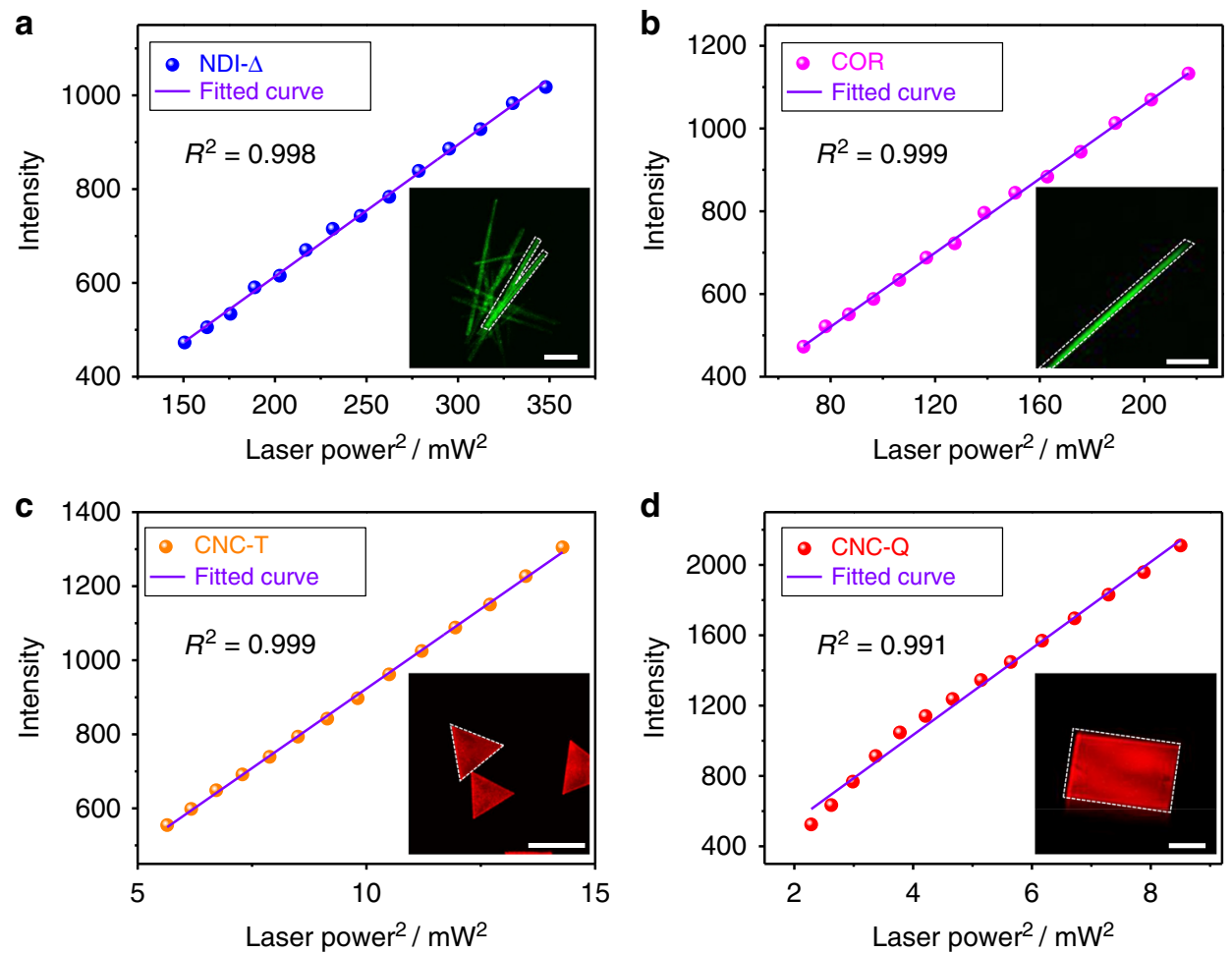

Fig. 7 Excitation power dependence of upconversion fluorescence intensity. The linear dependence between the upconversion fluorescence intensity and the square of the excitation laser power for a NDI- $\boldsymbol{\Delta}$ excited at $740 \mathrm{~nm}, \mathbf{b}$ COR excited at $740 \mathrm{~nm}, \mathbf{c}$ CNC-T excited at $1000 \mathrm{~nm}$ and $\mathbf{~ d ~ C N C - Q}$ excited at $1000 \mathrm{~nm}$. The upconversion fluorescence intensity of each material was collected from the marked region in the inset two-photon microscopy image. Scale bars: $20 \mu \mathrm{m}$ in (a, b, d), $50 \mu \mathrm{m}$ in (c).
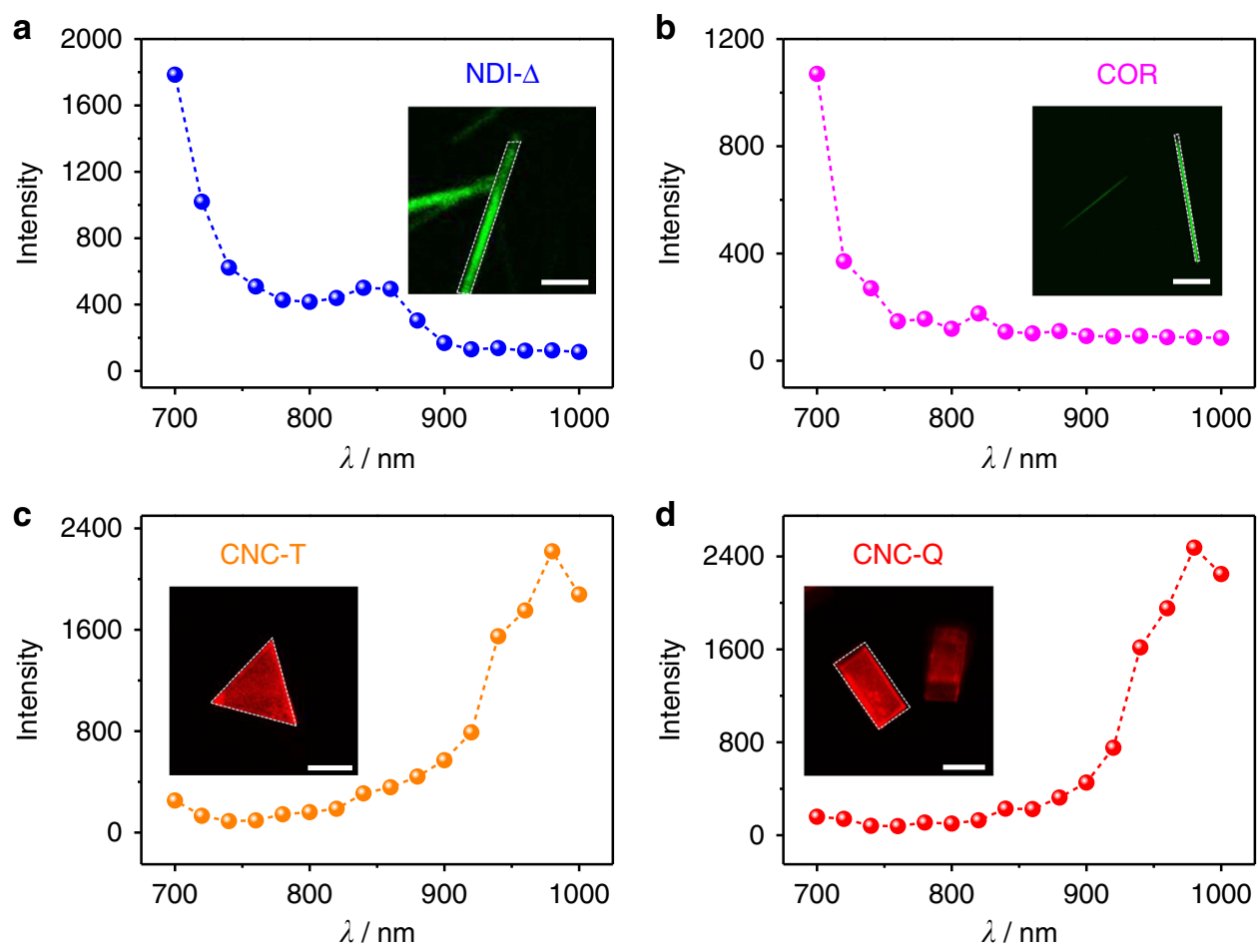

Fig. 8 Excitation wavelength dependence of upconversion fluorescence intensity. Two-photon excited spectra of a NDI- $\mathbf{\Delta}, \mathbf{b}$ COR, c CNC-T and d CNC-Q. The laser powers corresponding to the spectra were set to be $13.3,2.7,2.7$, and $2.7 \mathrm{~mW}$, respectively. The upconversion fluorescence intensity of each material was collected from the marked region in the inset two-photon microscopy image. Scale bars: $10 \mu \mathrm{m}$ in (a), $100 \mu \mathrm{m}$ in (b), $20 \mu \mathrm{m}$ in (c, d). 

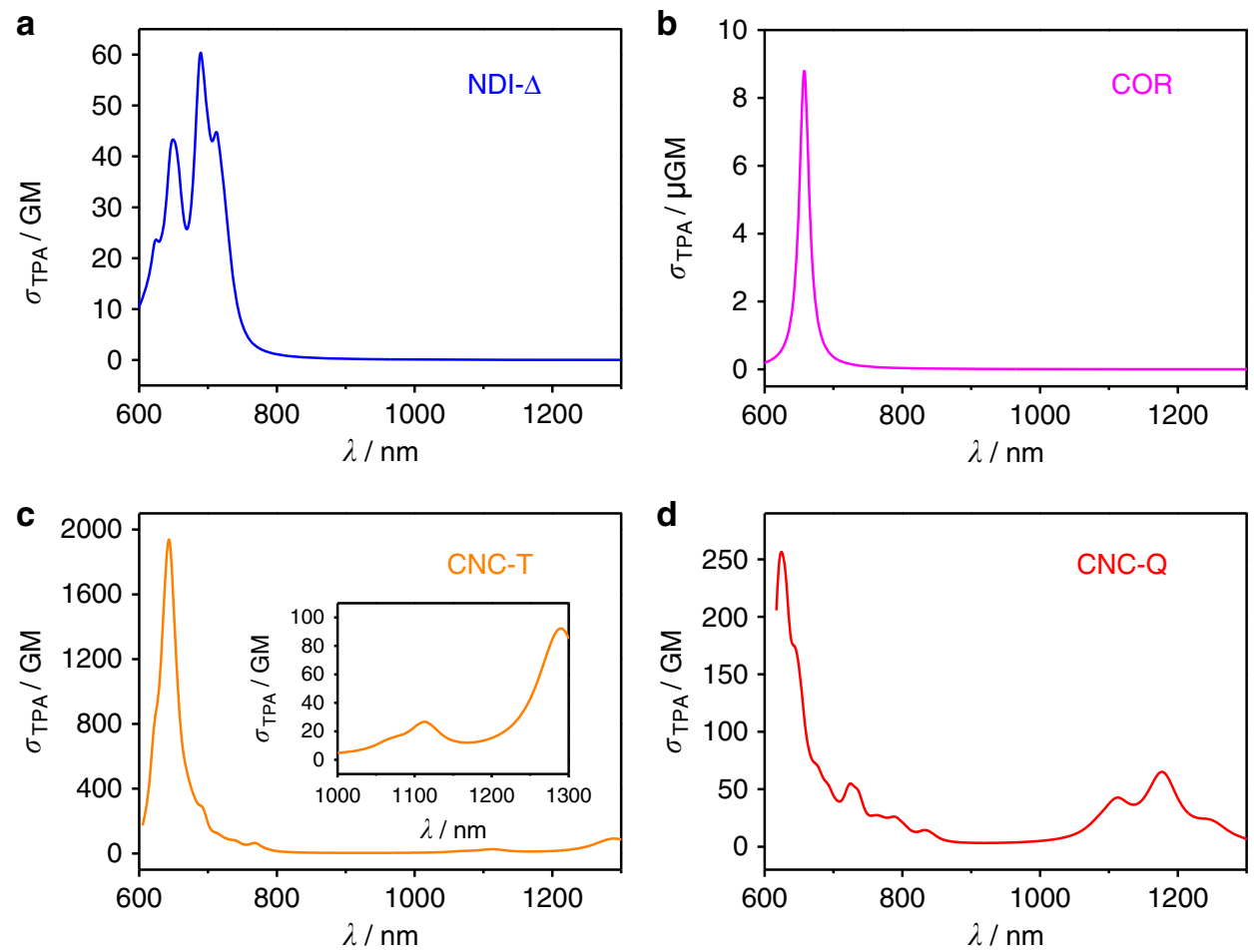

Fig. 9 Second linear response TDDFT calculations. The simulated two-photon excited spectra of a NDI- $\mathbf{\Delta}, \mathbf{b}$ COR, $\mathbf{c}$ CNC-T and $\mathbf{d}$ CNC-Q. The inset in c displays a magnified simulated two-photon excited spectrum of CNC-T in the region of 1000-1300 nm.

the nature of the solvents and the donor-acceptor stoichiometries. These co-crystals provide an example of how to gain better insights, through experiments supported by theory, into the relationship between the superstructures of materials and their properties. By altering the donor-acceptor ratio from 1:2 (CNCT) to 1:1 (CNC-Q), the intermolecular charge-transfer interactions become stronger and the energy bandgap becomes narrower in CNC-Q. Hence CNC-Q exhibits deep-red and NIR emission centered on $668 \mathrm{~nm}$, while CNC-T emits deep-red fluorescence with peak at $638 \mathrm{~nm}$. The TPA properties of the co-crystals are enhanced compared to those of the individual molecular crystals because of the intermolecular charge-transfer interactions. The CNC-T and CNC-Q co-crystals, not only exhibit red-shifted TPA spectra in the NIR-II region, but also possess larger TPA crosssections. Notably, the CNC-Q co-crystal emerges as an innovative two-photon excited NIR-emissive supramolecular material. The co-crystallization strategy presented here possesses the distinct advantage that comes with noncovalent synthesis, providing as it does a facile modular approach to advanced smart materials with multiphoton absorption and tunable emission properties.

\section{Methods}

Materials. All chemicals and solvents were purchased from commercial companies and used directly without further purification. (RRRRRR)-NDI- $\Delta-$ namely NDI- $\Delta$ employed in this research-was synthesized and purified following methods ${ }^{41}$ as shown in Supplementary Fig. 1. Coronene $\left(\mathrm{C}_{24} \mathrm{H}_{12}, 97 \%\right)$, defined as COR, was purchased from Sigma-Aldrich. Nuclear magnetic resonance (NMR) spectra were recorded on a Agilent $500 \mathrm{MHz}$ spectrometer with a working frequency of 500 $\mathrm{MHz}$ in the case of ${ }^{1} \mathrm{H}$ NMR spectroscopy. Chemical shifts are reported in ppm relative to the signals corresponding to the residual non-deuterated solvents $\left(\mathrm{CDCl}_{3}: \delta 7.26 \mathrm{ppm}\right)$.

Preparation of co-crystal CNC-T. NDI- $\Delta(20.8 \mathrm{mg}, 20 \mu \mathrm{mol})$ was first of all mixed with COR $(3.0 \mathrm{mg}, 10 \mu \mathrm{mol})$ in THF $(20 \mathrm{~mL})$ and dissolved by ultrasonic treatment (Supplementary Fig. 9). The solution was filtered with a $0.22-\mu \mathrm{m}$ syringe filter to remove insoluble impurities. With slow vapor diffusion of $\mathrm{MeOH}$ into the filtered solution, high quality red triangle-shaped co-crystals formed after one week.
Preparation of co-crystal CNC-Q. NDI- $\Delta(20.8 \mathrm{mg}, 20 \mu \mathrm{mol})$ was first of all mixed with $\mathrm{COR}(6.0 \mathrm{mg}, 20 \mu \mathrm{mol})$ in $\mathrm{CH}_{2} \mathrm{Cl}_{2}(20 \mathrm{~mL})$ and dissolved by ultrasonic treatment (Supplementary Fig. 10). The solution was filtered with a $0.22-\mu \mathrm{m}$ syringe filter to remove the insoluble impurities. With slow vapor diffusion of $\mathrm{Et}_{2} \mathrm{O}$ into the filtered solution, high quality red quadrangle-shaped co-crystals formed after one week.

Optical and fluorescence microscopies. Optical and fluorescence microscopy images were obtained at room temperature with a Nikon LV150, which was equipped with a high-intensity white light LED source and a 5 mega pixel color camera for image acquisition. Optical microscopy pictures were captured in the bright field mode. Fluorescence microscopy images were captured in the fluorescence mode.

Powder X-ray diffraction analysis. Powder X-ray diffraction (PXRD) was carried out on a STOE STADI MP powder diffractometer, which was equipped with an asymmetric curved Germanium monochromator $\left(\mathrm{Cu} K_{\alpha 1}\right.$ radiation, $\left.\lambda=1.54056 \AA\right)$ and a one-dimensional silicon strip detector (MYTHEN2 $1 \mathrm{~K}$ from DECTRIS). The line focused $\mathrm{Cu}$ X-ray tube was operated at $40 \mathrm{kV}$ and $40 \mathrm{~mA}$. Samples of micro/ nano-crystals were grown on glass substrates. NDI- $\Delta$ crystals were prepared by slow evaporation from a $\mathrm{PhCl}$ solution. COR crystals were prepared by slow evaporation from a $\mathrm{CH}_{2} \mathrm{Cl}_{2}$ solution. CNC-T was prepared by slow vapor diffusion of $\mathrm{MeOH}$ into a THF solution with a 1:2 donor-acceptor molar ratio. CNC-Q was prepared by slow vapor diffusion of $\mathrm{Et}_{2} \mathrm{O}$ into a $\mathrm{CH}_{2} \mathrm{Cl}_{2}$ solution with a 1:1 donor-acceptor molar ratio. Paratone oil was used to protect the crystals. Experiments were carried out in reflection geometry mode at room temperature. The simulated powder XRD patterns with $\mathrm{Cu} K_{\alpha 1}$ radiation were carried out by Mercury software 4.3.1.

Photophysical characterization. Solution UV-Vis absorption spectra were measured on a UV-3600 Shimadzu spectrophotometer at room temperature. Solution fluorescence spectra were recorded on a HORIBA FluoroMax-4 spectrometer at room temperature. Solid UV-Vis absorption spectra were measured in reflection mode at room temperature on a Shimadzu 3600 UV-visible-NIR spectro-

photometer equipped with an integrating sphere. Solid fluorescence spectra were performed with a HORIBA Fluorolog-3 spectrometer. Fluorescence lifetime measurements and solid-state absolute photoluminescence quantum yields were obtained using a HORIBA FluoroMax-4 spectrometer equipped with an integrating sphere (HORIBA Quanta- $\varphi$ ).

Raman spectroscopy. Raman spectra were carried out on a HORIBA LabRAM HR Evolution Confocal Raman instrument. The system was equipped with (i) 
multi-laser (473, 532, 633, and $785 \mathrm{~nm}$ ), (ii) a confocal microscope for high spatial resolution, (iii) a Raman spectrometer detector for high spectral resolution and wide spectral range analysis, (iv) a high-precision DuoScan imaging instrument and (v) an electron multiplying CCD in order to enhance the measured S/N ratios.

Cyclic voltammetry. Cyclic voltammetry (CV) was performed on a Gamry Multipurpose instrument (Reference 600) interfaced to a PC under $\mathrm{N}_{2}$ atmosphere at $298 \mathrm{~K}$. The CV experiments were recorded with a glassy carbon working electrode $\left(0.071 \mathrm{~cm}^{2}\right.$, Cypress system). The electrode surface was polished routinely with alumina-water slurry on a felt surface before use. The counter electrode was a Pt coil, and the reference electrode was $\mathrm{Ag} / \mathrm{AgCl}$. CV Experiments were carried out in a $0.1 \mathrm{M}$ solution of $\left[\mathrm{Bu}_{4} \mathrm{~N}\right]\left[\mathrm{PF}_{6}\right]$ electrolyte in $\mathrm{CH}_{2} \mathrm{Cl}_{2}$ at a $100 \mathrm{mV} / \mathrm{s}$ scan rate. The sample concentration was $0.5 \mathrm{mM}$.

Two-photon microscopy. The upconversion emission and TPA-related measurements were performed on a Nikon A1R-MP + Multiphoton Microscope with penetration depth up to $1.5 \mathrm{~mm}$. The microscope was equipped with a tunable Chameleon Vision titanium sapphire laser from 700 to $1000 \mathrm{~nm}$. In addition, the GaAsP detectors were applied for increased sensitivity. The resonant scanner was capable of scanning up to $\sim 400$ frames per second.

Density functional theory computational methods. Structures for the singlemolecule calculations were obtained from X-ray single crystal diffraction data. All electron density functional theory (DFT) single point calculations were performed at the B3LYP/TZP level in the Amsterdam Density Functional program (ADF, version 2018.104) ${ }^{60-62}$. The orbital surfaces were visualized with ADFView.

The TPA spectra were computed by second linear response TDDFT (SLRTDDFT), an in-house code implemented within the NWChem suite ${ }^{63}$. This theory computes transition dipoles between different excited states with a computational cost similar to that of traditional linear-response (LR) TDDFT calculations. SLR-TDDFT Calculations can be performed by either "relaxing" the orbitals of the system, or by utilizing an unrelaxed scheme in which the excited-state transition dipoles are determined by a simple numerical differentiation procedure ${ }^{55}$. Given the size of the molecules, in this work the unrelaxed method was used on account of its computational efficiency. In principle, the unrelaxed method is equivalent to employing the auxiliary Casida wave functions ${ }^{64}$, but they can be reliably accurate ${ }^{65}$. For these calculations, the exchange-correlation functional LDA0 was used ${ }^{66}$. This functional-for optical properties-is as accurate as B3LYP, and it provides an additional speed-up for the calculations, which is critical in this case, given the relatively large number of atoms and excited states, especially for CNC-Q. The SLRTDDFT requires as a first step a regular LR-TDDFT calculation under the TammDancoff approximation. Also, for computational convenience, the double- $\zeta$ basis set and effective-core potential SBKJC were applied. The Davidson convergence threshold is $10^{-3}$ for COR and NDI- $\Delta$, and $10^{-2}$ for CNC-T and CNC-Q. This requirement guarantees the energies are accurate up to a fraction of a meV. In the LR-TDDFT calculation, we neglect virtual orbitals with energies above 12 and $8 \mathrm{eV}$ for CNC-T and CNC-Q, respectively. These ranges are sufficient to cover the TPA spectra: the excited state with largest energy is below $4.14 \mathrm{eV}$. The SLR-TDDFT algorithm provides all the transition dipoles required to compute the TPA cross-section as a function of the photon wavelength. We assume random circularly polarized light interaction. The frequency-dependent TPA cross-section was computed using the formula for this type of light interaction ${ }^{67,68}$. We assume a Lorentzian line shape function, a broadening factor of $0.05 \mathrm{eV}$ for virtual states, and $0.1 \mathrm{eV}$ for the final states.

\section{Data availability}

All the data supporting the conclusions are included in this article and its Supplementary files, or are available from the authors upon reasonable request. The single-crystal diffraction data for NDI- $\Delta, \mathbf{C N C}-\mathrm{T}$ and $\mathbf{C N C}-\mathrm{Q}$ have all been deposited in the Cambridge Crystallographic Data Centre (CCDC). The deposition numbers are CCDC 2004158-2004160, respectively.

\section{Code availability}

The two-photon absorption post-processing code used in this article is available at https://gitlab.com/plusxmartin/insomnia.

Received: 18 May 2020; Accepted: 21 August 2020;

Published online: 15 September 2020

\section{References}

1. Antaris, A. L. et al. A small-molecule dye for NIR-II imaging. Nat. Mater. 15, 235-242 (2016).

2. Sreejith, S. et al. Near-infrared squaraine dye encapsulated micelles for in vivo fluorescence and photoacoustic bimodal imaging. ACS Nano 9, 5695-5704 (2015).
3. Yang, Q. et al. Donor engineering for NIR-II molecular fluorophores with enhanced fluorescent performance. J. Am. Chem. Soc. 140, 1715-1724 (2018).

4. Chen, Q. et al. Photothermal therapy with immune-adjuvant nanoparticles together with checkpoint blockade for effective cancer immunotherapy. Nat. Commun. 7, 13193 (2016)

5. Jung, H. S. et al. Organic molecule-based photothermal agents: an expanding photothermal therapy universe. Chem. Soc. Rev. 47, 2280-2297 (2018)

6. Sun, Y. et al. Melanin-dot-mediated delivery of metallacycle for NIR-II/ photoacoustic dual-modal imaging-guided chemo-photothermal synergistic therapy. Proc. Natl Acad. Sci. USA 116, 16729-16735 (2019).

7. Jalani, G., Tam, V., Vetrone, F. \& Cerruti, M. Seeing, targeting and delivering with upconverting nanoparticles. J. Am. Chem. Soc. 140, 10923-10931 (2018).

8. Ly, K. T. et al. Near-infrared organic light-emitting diodes with very high external quantum efficiency and radiance. Nat. Photonics 11, 63-68 (2017).

9. Yang, Y., Hughes, R. P. \& Aprahamian, I. Near-infrared light activated Azo$\mathrm{BF}_{2}$ switches. J. Am. Chem. Soc. 136, 13190-13193 (2014).

10. Zheng, Z. et al. Aggregation-induced nonlinear optical effects of AIEgen nanocrystals for ultradeep in vivo bioimaging. Adv. Mater. 31, 1904799 (2019).

11. Yuan, L., Lin, W., Zheng, K., He, L. \& Huang, W. Far-red to near infrared analyte-responsive fluorescent probes based on organic fluorophore platforms for fluorescence imaging. Chem. Soc. Rev. 42, 622-661 (2013).

12. Dou, L., Liu, Y., Hong, Z., Li, G. \& Yang, Y. Low-bandgap near-IR conjugated polymers/molecules for organic electronics. Chem. Rev. 115, 12633-12665 (2015).

13. Li, Y. et al. Design of AIEgens for near-infrared IIb imaging through structural modulation at molecular and morphological levels. Nat. Commun. 11, 1255 (2020).

14. Zampetti, A., Minotto, A., Cacialli, F. \& Near-infrared (NIR) organic lightemitting diodes (OLEDs): challenges and opportunities. Adv. Funct. Mater. 29, 1807623 (2019).

15. Wan, H. et al. A bright organic NIR-II nanofluorophore for three-dimensional imaging into biological tissues. Nat. Commun. 9, 1171 (2018).

16. Kim, H. M. \& Cho, B. R. Small-molecule two-photon probes for bioimaging applications. Chem. Rev. 115, 5014-5055 (2015).

17. Sun, C.-L. et al. Rational design of organic probes for turn-on two-photon excited fluorescence imaging and photodynamic therapy. Chem 5, 600-616 (2019).

18. Zhou, Z. et al. A self-assembled Ru-Pt metallacage as a lysosome-targeting photosensitizer for 2-photon photodynamic therapy. Proc. Natl Acad. Sci. USA 116, 20296-20302 (2019).

19. Carlotti, M. \& Mattoli, V. Functional materials for two-photon polymerization in microfabrication. Small 15, 1902687 (2019).

20. Yu, J. et al. Two-photon responsive metal-organic framework. J. Am. Chem. Soc. 137, 4026-4029 (2015).

21. Shao, B. et al. Solution and solid-state emission toggling of a photochromic hydrazone. J. Am. Chem. Soc. 140, 12323-12327 (2018).

22. Yu, Y. et al. Transformation from nonlasing to lasing in organic solid-state through the cocrystal engineering. ACS Photonics 6, 1798-1803 (2019).

23. Catalano, L. et al. Rotational dynamics of diazabicyclo[2.2.2]octane in isomorphous halogen-bonded co-crystals: entropic and enthalpic effects. $J$. Am. Chem. Soc. 139, 843-848 (2017).

24. Jiang, X. et al. Thermally activated transient dipoles and rotational dynamics of hydrogen-bonded and charge-transferred diazabicyclo [2.2.2] octane molecular rotors. J. Am. Chem. Soc. 141, 16802-16809 (2019).

25. Jiang, H. et al. Tuning of the degree of charge transfer and the electronic properties in organic binary compounds by crystal engineering: a perspective. J. Mater. Chem. C. 6, 1884-1902 (2018).

26. Huang, Y., Wang, Z., Chen, Z. \& Zhang, Q. Organic cocrystals: beyond electrical conductivities and field-effect transistors (FETs). Angew. Chem. Int. Ed. 58, 9696-9711 (2019).

27. Park, S. K. et al. Highly luminescent 2D-type slab crystals based on a molecular charge-transfer complex as promising organic light-emitting transistor materials. Adv. Mater. 29, 1701346 (2017).

28. Catalano, L. et al. Dynamic characterization of crystalline supramolecular rotors assembled through halogen bonding. J. Am. Chem. Soc. 137, 15386-15389 (2015).

29. Yang, Y.-D. et al. Time-dependent solid-state molecular motion and colour tuning of host-guest systems by organic solvents. Nat. Commun. 11, 77 (2020).

30. Goetz, K. P. et al. Charge-transfer complexes: new perspectives on an old class of compounds. J. Mater. Chem. C. 2, 3065-3076 (2014).

31. Tayi, A. S. et al. Room-temperature ferroelectricity in supramolecular networks of charge-transfer complexes. Nature 488, 485-489 (2012).

32. Zhuo, M.-P. et al. Hierarchical self-assembly of organic heterostructure nanowires. Nat. Commun. 10, 3839 (2019).

33. Yan, D. et al. A cocrystal strategy to tune the luminescent properties of stilbene-type organic solid-state materials. Angew. Chem. Int. Ed. 50, 12483-12486 (2011).

34. Zhang, J., Xu, W., Sheng, P., Zhao, G. \& Zhu, D. Organic donor-acceptor complexes as novel organic semiconductors. Acc. Chem. Res. 50, 1654-1662 (2017). 
35. Ye, $\mathrm{X}$. et al. $1 \mathrm{D}$ versus $2 \mathrm{D}$ cocrystals growth via microspacing in-air sublimation. Nat. Commun. 10, 761 (2019).

36. Liu, Y. et al. Piezochromic luminescence of donor-acceptor cocrystals: Distinct responses to anisotropic grinding and isotropic compression. Angew. Chem. Int. Ed. 57, 15670-15674 (2018).

37. Bai, L. et al. Halogen-assisted piezochromic supramolecular assemblies for versatile haptic memory. J. Am. Chem. Soc. 139, 436-441 (2017).

38. Liu, G. et al. Self-healing behavior in a thermo-mechanically responsive cocrystal during a reversible phase transition. Angew. Chem. Int. Ed. 56, 198-202 (2017).

39. Wang, Y. et al. Cocrystals strategy towards materials for near-infrared photothermal conversion and imaging. Angew. Chem. Int. Ed. 57, 3963-3967 (2018).

40. Bolton, O., Lee, K., Kim, H. J., Lin, K. Y. \& Kim, J. Activating efficient phosphorescence from purely organic materials by crystal design. Nat. Chem. 3, 205-210 (2011).

41. Schneebeli, S. T. et al. Electron sharing and anion $-\pi$ recognition in molecular triangular prisms. Angew. Chem. Int. Ed. 52, 13100-13104 (2013).

42. Liu, Z. et al. Assembly of supramolecular nanotubes from molecular triangles and 1,2-dihalohydrocarbons. J. Am. Chem. Soc. 136, 16651-16660 (2014).

43. Beldjoudi, Y. et al. Supramolecular tessellations by a rigid naphthalene diimide triangle. J. Am. Chem. Soc. 141, 17783-17795 (2019).

44. Chen, D. et al. A rigid naphthalenediimide triangle for organic rechargeable lithium-ion batteries. Adv. Mater. 27, 2907-2912 (2015).

45. Xiao, J. et al. Preparation, characterization, and photoswitching/light-emitting behaviors of coronene nanowires. J. Mater. Chem. 21, 1423-1427 (2011).

46. Aryanpour, K. et al. Subgap two-photon states in polycyclic aromatic hydrocarbons: evidence for strong electron correlations. J. Phy. Chem. C. 118, 3331-3339 (2014).

47. Yan, D. \& Evans, D. G. Molecular crystalline materials with tunable luminescent properties: from polymorphs to multi-component solids. Mater. Horiz. 1, 46-57 (2014)

48. Salzillo, T. et al. Structure, stoichiometry, and charge transfer in cocrystals of perylene with TCNQ-Fx. Cryst. Growth Des. 16, 3028-3036 (2016).

49. Sun, L., Wang, Y., Yang, F., Zhang, X. \& Hu, W. Cocrystal engineering: a collaborative strategy toward functional materials. Adv. Mater. 31, 1902328 (2019).

50. Würthner, F., Kaiser, T. E. \& Saha-Möller, C. R. J-aggregates: from serendipitous discovery to supramolecular engineering of functional dye materials. Angew. Chem. Int. Ed. 50, 3376-3410 (2011).

51. Gernert, M. et al. Cyclic (amino)(aryl)carbenes enter the field of chromophore ligands: Expanded $\pi$ system leads to unusually deep red emitting $\mathrm{CuI}$ compounds. J. Am. Chem. Soc. 142, 8897-8909 (2020).

52. Chen, H., Dong, B., Tang, Y. \& Lin, W. A unique "integration" strategy for the rational design of optically tunable near-infrared fluorophores. Acc. Chem. Res. 50, 1410-1422 (2017).

53. Pawlicki, M., Collins, H. A., Denning, R. G. \& Anderson, H. L. Two-photon absorption and the design of two-photon dyes. Angew. Chem. Int. Ed. 48 3244-3266 (2009).

54. Drobizhev, M., Makarov, N. S., Tillo, S. E., Hughes, T. E. \& Rebane, A. Twophoton absorption properties of fluorescent proteins. Nat. Methods 8, 393-399 (2011).

55. Mosquera, M. A., Chen, L. X., Ratner, M. A. \& Schatz, G. C. Sequential double excitations from linear-response time-dependent density functional theory. $J$. Chem. Phys. 144, 204105 (2016).

56. Mosquera, M. A. et al. Exciton absorption spectra by linear response methods: application to conjugated polymers. J. Am. Chem. Soc. 139, 3728-3735 (2017).

57. Gao, S. et al. Albumin tailoring fluorescence and photothermal conversion effect of near-infrared-II fluorophore with aggregation-induced emission characteristics. Nat. Commun. 10, 2206 (2019).

58. Qi, J. et al. Aggregation-induced emission luminogen with near-infrared-II excitation and near-infrared-I emission for ultradeep intravital two-photon microscopy. ACS Nano 12, 7936-7945 (2018).

59. Sun, L. et al. Intermolecular charge-transfer interactions facilitate two-photon absorption in styrylpyridine-tetracyanobenzene cocrystals. Angew. Chem. Int. Ed. 56, 7831-7835 (2017).

60. Versluis, L. \& Ziegler, T. The determination of molecular structures by density functional theory. The evaluation of analytical energy gradients by numerical integration. J. Chem. Phys. 88, 322-328 (1988).

61. Te Velde, G. et al. Chemistry with ADF. J. Comput. Chem. 22, 931-967 (2001).

62. Van Lenthe, E. \& Baerends, E. J. Optimized slater-type basis sets for the elements 1-118. J. Comput. Chem. 24, 1142-1156 (2003).

63. Valiev, M. et al. NWChem: a comprehensive and scalable open-source solution for large scale molecular simulations. Comput. Phys. Commun. 181, 1477 (2010).

64. Tavernelli, I., Curchod, B. F. E. \& Rothlisberger, U. On nonadiabatic coupling vectors in time-dependent density functional theory. J. Chem. Phys. 131, 196101 (2009).
65. Sheng, X. et al. Excited-state absorption by linear response time-dependent density functional theory. J. Phy. Chem. C. 124, 4693-4700 (2020).

66. Mosquera, M. A., Borca, C. H., Ratner, M. A. \& Schatz, G. C. Connection between hybrid functionals and importance of the local density approximation. J. Phys. Chem. A 120, 1605-1612 (2016).

67. Silva, D. L., Krawczyk, P., Bartkowiak, W. \& Mendonça, C. R. Theoretical study of one- and two-photon absorption spectra of azoaromatic compounds. J. Chem. Phys. 131, 244516 (2009).

68. Ohta, K., Antonov, L., Yamada, S. \& Kamada, K. Theoretical study of the twophoton absorption properties of several asymmetrically substituted stilbenoid molecules. J. Chem. Phys. 127, 084504 (2007).

\section{Acknowledgements}

The authors thank Northwestern University (NU) for its support of this research. The authors would like to thank Professor Justin Notestein and Mark Taylor for technical support on solid-state UV-Vis absorption spectroscopy measurements. The A1R-MP+ multiphoton microscope was acquired through an S10 shared instrumentation grant awarded to Teng-Leong Chew (1 S10 OD010398-01). This work made use of the NUFAB facility of Northwestern University's NUANCE Center, NNCI-SHyNE Resource (NSF ECCS-1542205) and the MRSEC program (NSF DMR-1720139) at the Materials Research Center. L.O.J. and G.C.S. acknowledge support from NSF grant CHE-1836392 (two-photon applications); M.A.M., G.C.S., and M.A.R. acknowledge support from the Department of Energy, grant DE-AC02-06CH11357 (functional materials and spectroscopy applications), and grant DE-SC0004752 (theory development). This research was also supported in part through the computational resources and staff contributions provided for the Quest high performance computing facility at Northwestern University which is jointly supported by the Office of the Provost, the Office for Research, and Northwestern University Information Technology. S.C. and M.R.W. thank the support from the U.S Department of Energy, Office of Science, Office of Basic Energy Sciences under Award DEFG02-99ER14999.

\section{Author contributions}

Y.W. and H.W. conceived the research, and carried out the major experiments and analyses. P.L. prepared the macrocycle materials. S.C. and M.R.W. carried out the solid state fluorescence measurements. L.O.J., M.A.M., M.A.R., and G.C.S. conducted DFT and TDDFT calculations. C.L.S. did the X-ray crystallographic analysis. Y.W. and H.W wrote the paper. L.Z., K.C., H.C., and X.C. were involved in the discussions and contributed to the manuscript preparation. J.F.S. directed and supervised the research. All authors discussed the experimental results and contributed to the preparation of the paper.

\section{Competing interests}

The authors declare no competing interests.

\section{Additional information}

Supplementary information is available for this paper at https://doi.org/10.1038/s41467020-18431-7.

Correspondence and requests for materials should be addressed to J.F.S.

Peer review information Nature Communications thanks Mirella Del Zoppo, Bing Yang and the other, anonymous, reviewer(s) for their contribution to the peer review of this work.

\section{Reprints and permission information is available at http://www.nature.com/reprints}

Publisher's note Springer Nature remains neutral with regard to jurisdictional claims in published maps and institutional affiliations.

pen Access This article is licensed under a Creative Commons Attribution 4.0 International License, which permits use, sharing, adaptation, distribution and reproduction in any medium or format, as long as you give appropriate credit to the original author(s) and the source, provide a link to the Creative Commons license, and indicate if changes were made. The images or other third party material in this article are included in the article's Creative Commons license, unless indicated otherwise in a credit line to the material. If material is not included in the article's Creative Commons license and your intended use is not permitted by statutory regulation or exceeds the permitted use, you will need to obtain permission directly from the copyright holder. To view a copy of this license, visit http://creativecommons.org/ licenses/by/4.0/.

(C) The Author(s) 2020 